\title{
New Phase Sequences in Selected Mapping Scheme for Peak to Average Power Ratio Reduction in Orthogonal Frequency Division Multiplexing
}

\author{
Abhinav V. Deshpande \\ Internal Full Time (IFT) Ph. D. Research Scholar, School of Electronics Engineering (SENSE) \\ Vellore Institute of Technology (VIT), Vellore, Tamil Nadu, India-632014 \\ avd.a.deshpande@gmail.com
}

\begin{abstract}
Orthogonal Frequency Division Multiplexing (OFDM) is a robust and effective multicarrier transmission technique for high speed communication in the wireless mobile environment and applications. A major challenging issue in the application of OFDM is its high peak to average ratio (PAPR). The Selective Mapping (SLM) is one of the promising techniques which offer a distortion less PAPR reduction at the cost of bandwidth efficiency and computational complexity. The rows of the Hadamard, Riemann and the Centering matrices are suggested to be used as the phase sequences in the literature. In this research work, the original SLM scheme is modified by using the new phase sequences, which are the combinations of the circulating matrix along with the Hadamard and the Riemann matrices. The simulation results show that the proposed phase sequences offer an improved PAPR reduction and thus outperform some of the previously proposed techniques.
\end{abstract}

Keywords - Orthogonal Frequency Division Multiplexing (OFDM); Peak to Average Power Ratio (PAPR); Selected Mapping (SLM); Hadamard Code; Reed-Solomon Code

\section{Introduction}

The Orthogonal Frequency Division Multiplexing (OFDM) is widely implemented in various high-speed wireless communication standards because of its favorable properties such as the high spectral efficiency, robustness to the channel fading, immunity to the impulse interference and the capability of handling the multipath fading. Interestingly, the OFDM is a combination of the modulation and multiplexing. This technique is a multicarrier modulation technique, which employs several carriers, within the allocated bandwidth, in order to convey the information from the source to the destination. Each carrier may employ one of the several available digital modulation techniques (Binary Phase Shift Keying (BPSK), Quadrature Phase Shift Keying (QPSK) or the Quadrature Amplitude Modulation (QAM)). Generally, an OFDM signal can be represented as follows:

$\mathrm{C}(\mathrm{t})=\Sigma_{\mathrm{n}=0}{ }^{\mathrm{N}-1} \mathrm{~S}_{\mathrm{n}}(\mathrm{t}) \sin \left(2 \pi \mathrm{f}_{\mathrm{n}} \mathrm{t}\right)$

Eqn. (1)

Where, $S_{n}(t)=$ the symbols which are mapped to the chosen constellation (BPSK/QPSK/QAM)

$\mathrm{f}_{\mathrm{n}}$ represents the orthogonal frequencies.

Equation (1) can be thought of as an Inverse Fast Fourier Transform (IFFT) where " $\mathrm{N}$ " is the size of the IFFT. The Fourier transform breaks a signal into different frequency bins by multiplying the signal with a series of sinusoids. Since the OFDM signal [c (t) in the Equation (1)] is in the time domain, the IFFT is the appropriate choice to use at the transmitter, which can also be interpreted as converting the frequency domain samples to the time domain sample. In order to acquire the original transmitted signal, the FFT is performed at the receiver side [2].

\section{Peak to Average Power Ratio}

The OFDM has several features which make it an attractive modulation scheme for the high-speed transmission links. However, one major limitation is its large peak to average power ratio (PAPR). These large peaks cause a saturation in the power amplifiers at the transmitting end, leading to the inter modulation among the subcarriers, which causes an increase in the out of band (OOB) energy. Hence, in order to design a cost effective and a robust system, it is highly desirable to reduce the PAPR. The mathematical representation of the PAPR of an OFDM signal can be given as below:

PAPR $\{\mathrm{c}(\mathrm{t})\}=\max 0 \leq \mathrm{n} \leq \mathrm{N}-1|\mathrm{c}(\mathrm{t})|^{2} / \mathrm{E}\left[|\mathrm{c}(\mathrm{t})|^{2}\right]$ Eqn. (2) Where, $\mathrm{E}\{$.$\} denotes the expectation.$

The PAPR can be expressed in "db" as follows, PAPR $(\mathrm{db})=10 \log _{10}$ [PAPR ( c )] Eqn. (3) Some of the PAPR reduction schemes are clipping and filtering, companding, coding, selective mapping (SLM), partial transmit sequence (PTS), tone reservation, tone rejection, constellation extension. The SLM technique improves the PAPR statistics of an OFDM signal significantly without any in-band distortion and the out-ofband radiation. The selection of a proper phase sequences in order to achieve a good PAPR reduction is very important in the SLM technique [3].

\section{Selected Mapping Technique}

The Selected Mapping (SLM) method is a distortion less probabilistic technique for the purpose of PAPR 
reduction. This technique is called as distortion less because the quantity with which the actual signal is altered is sent as the side information [4]. In this method, the original modulated OFDM data block is multiplied element by element with a phase sequence:

$\mathrm{B}^{(\mathrm{u})}=\left[\mathrm{b}_{\mathrm{u}, 0}, \mathrm{~b}_{\mathrm{u}, 1}, \ldots, \mathrm{b}_{\mathrm{u}, \mathrm{N}-1}\right]^{\mathrm{T}} ; \quad \mathrm{u}=0,1,2, \ldots, \mathrm{U} \quad$ Eqn. (4) In order to make the $\mathrm{U}$ phase rotated OFDM data blocks $\mathrm{X}^{(\mathrm{u})}=\left[\mathrm{X}_{\mathrm{u}, 0}, \mathrm{X}_{\mathrm{u}, 1}, \ldots, \mathrm{X}_{\mathrm{u}, \mathrm{N}-1}\right]^{\mathrm{T}}$

Where, $X_{u, m}=X_{m} * b_{u, m} ; m=0,1, \ldots . ., N-1$

All the phase rotated OFDM data blocks represent the same information as that of the unmodified OFDM. The PAPR is calculated for the phase rotated OFDM data blocks by using Eqn. (2) and (3). Among the modified data blocks, one with the lowest PAPR is selected and transmitted. The information about the selected phase sequence should be transmitted to the receiver as the side information. The reverse operation should be performed at the receiver in order to obtain the original data block [3][4].

\section{Different Phase Sequences}

The classical definition of the SLM refers to a random sequence to be used as the phase altering sequence. Accordingly, the complete phase sequence should be sent as the side information in a conventional SLM. If the rows of known special matrices are used as the phase altering sequence, then the side information can be greatly reduced. These special matrices can be generated at the receiver and only the address (row index) of the selected phase sequence can be sent as the side information [5][6]. The following are some of the matrices which can be used to generate the phase altering sequences:

\subsection{Review Stage}

The Hadamard matrices are the square matrices whose entries are +1 or -1 , and whose rows and the columns are mutually orthogonal.

$\begin{array}{llll}\mathrm{H}_{1}=[1] & & & \text { Eqn.(7) } \\ \mathrm{H}_{2}{ }^{\mathrm{k}}=\left[\begin{array}{llll}\mathrm{H}_{2}{ }^{\mathrm{k}-1} & \mathrm{H}_{2}{ }^{\mathrm{k}-1} & \mathrm{H}_{2}{ }^{\mathrm{k}-1} & \mathrm{H}_{2}{ }^{\mathrm{k}-1}\end{array}\right]=\mathrm{H}_{2}{ }^{*} \mathrm{H}_{2}{ }^{\mathrm{k}-1} & \text { Eqn. (8) }\end{array}$

\subsection{Riemann Matrix}

The Riemann matrix ${ }^{\circledR}$ is obtained by removing the first row and first column of the matrix $A$, where

$$
\begin{aligned}
A(I, j) & =I-1 \text { if "I" divides " "j" } & & \text { Eqn. (9) } \\
& =1 \text { otherwise } & & \text { Eqn. (10) }
\end{aligned}
$$

\subsection{Centering Matrix}

The centering matrix is symmetric and idempotent, which when multiplied with a vector has the identical effect as subtracting the mean of the components of the vector from every component [7]. The structure of the centering matrix $\left(C_{n}\right)$ is given by the relationship:
$\mathrm{C}_{\mathrm{n}}=1_{\mathrm{n}}-1 / \mathrm{n} 0_{\mathrm{n}}$

Eqn. (11)

Where, $l_{n}$ is the $n$ by $n$ identity matrix and $0_{n}$ is an $n$ by $n$ matrix of all 1 's.

\section{New Phase Sequences}

The conventional definition of the SLM states the use of a random phase sequences for the purpose of PAPR reduction. Thus, there can be many possible phase sequences. On the contrary, instead of generating a random phase sequences, various special matrices are suggested in the latest reference literature [5][6][7]. The Hadamard and the Riemann matrices are preferred for the generation of the phase sequences. The Riemann matrix has been suggested to be the best for the purpose of PAPR reduction so far. Another matrix suggested recently is the Centering matrix. This matrix performs even better than the Riemann matrix [7]. In this experimentation, the combinations of the Centering matrix with the special matrices like the Hadamard and the Riemann are explored. One of the possible combinations is the Centered Hadamard and the Centered Riemann matrices. These matrices are generated by performing the Centering operation on the Hadamard and the Riemann matrices respectively that is the matrix multiplication takes place in between the Centering matrix and the Hadamard/Riemann matrix.

\subsection{Centering of Hadamard and Riemann Matrices}

The centering of a data matrix is done by multiplying it with the centering matrix. After the operation of centering, the values of the data matrix get close to the mean value [8].

$\mathrm{C}_{\mathrm{h}}=\mathrm{C}_{\mathrm{n}} * \mathrm{H}_{\mathrm{n}}$

Eqn. (12)

$\mathrm{C}_{\mathrm{r}}=\mathrm{C}_{\mathrm{n}} * \mathrm{R}_{\mathrm{n}}$

Eqn. (13)

Where,

$\mathrm{C}_{\mathrm{h}}=$ Centered Hadamard matrix ( $\mathrm{n}$ by $\mathrm{n}$ )

$\mathrm{C}_{\mathrm{r}}=$ Centered Riemann matrix ( $\mathrm{n}$ by $\mathrm{n}$ )

$\mathrm{H}_{\mathrm{n}}=$ Hadamard matrix ( $\mathrm{n}$ by $\mathrm{n}$ )

$\mathrm{C}_{\mathrm{n}}=$ Centering matrix ( $\mathrm{n}$ by $\mathrm{n}$ )

\subsection{Proposed Phase Sequence}

In the quest of finding a new phase sequences, another combination of centering matrix with the special matrices is formed. The proposed phase sequences are generated by performing element-by-element multiplication of the Centering matrix with the Hadamard and the Riemann matrices. This combination delivers a new set of phase sequences, which offers a better result than the other matrices which are considered in this experimentation in between the two matrices, it has a fewer computations than the Centered matrices which are explained earlier, instead of using any random sequence, a combination of two standard matrices is being used in this research work. This matrix combination can also be generated at the receiver, 
leading to a reduction in the side information, as only the row index number can be sent as the side information. The modifications in the existing SLM scheme are introduced by the new proposed phase sequences

$\mathrm{C}_{\text {ch (new) }}=\mathrm{C}_{\mathrm{n}} * \mathrm{H}_{\mathrm{n}}$

Eqn. (14)

$\mathrm{C}_{\text {cr (new) }}=\mathrm{C}_{\mathrm{n}} * \mathrm{R}_{\mathrm{n}}$

Eqn. (15)

\section{Side Information}

The side information is the major significant feature of the SLM technique. If the side information is not received correctly, then the effectively erroneous detection of the received signal takes place. Thus, it is highly essential to save the side information from getting a corrupted while it travels through the channel. The error control coding or the channel coding can be an effective measure to protect the side information. In this experimentation, the side information is sent in the form of a row index or the row number of the phase sequence generator matrix, which provides the least PAPR for a particular OFDM symbol [7][9]. The $\mathrm{BCH}$ coding is done on the set of the side information which is collected for all the corresponding OFDM symbols.

\section{BCH Codes}

The Bose, Chaudhuri and Hocquenghem $(\mathrm{BCH})$ codes form a large class of a powerful random error correcting cyclic codes. This class of codes is a remarkable generalization of the Hamming codes for multiple error correction [10]. For any positive integers $\mathrm{m}(\mathrm{m} \geq 3)$ and $\mathrm{t}(\mathrm{t}$ $<2^{\mathrm{m}-1}$ ), there exists a binary $\mathrm{BCH}$ code with the following parameters:

$\begin{array}{lll}\text { Block Length } & \mathrm{n}=2^{\mathrm{m}}-1 & \text { Eqn. (16) } \\ \text { Number of Parity Check Digits } & \mathrm{n}-\mathrm{k} \leq \mathrm{mt} & \text { Eqn. (17) } \\ \text { Minimum Distance } & \mathrm{d}_{\min } \geq 2 \mathrm{t}+1 & \text { Eqn. (18) }\end{array}$

This code is capable of correcting any combination of " $\mathrm{t}$ " or a fewer errors in a block of $\mathrm{n}=2^{\mathrm{m}}-1$ digit. This code is called as a t error correcting $\mathrm{BCH}$ code. The generator polynomial of this code is specified in terms of its roots from the Galois Field GF (2m) [11].

$\mathrm{A}(7,4) \mathrm{BCH}$ code is used in this experimentation. The $\mathrm{BCH}$ coded side information is separately the IFFTed by using a 8 point IFFT after applying four times oversampling. During transmission, the OFDM data block is sent first and the side information which is related to that particular data block follows on a separate set of frequencies.

\section{Simulation Results}

The following are the system specifications which are used for the unmodified OFDM, conventional SLM and the modified SLM scheme [12]. The simulation is performed by using the software tool which is known as MATLAB. The side information which is generated is of 4 bits $\left(\log _{2} \mathrm{U}\right)$ for every OFDM symbol. The CCDF plot which is shown in Figure 2 shows the PAPR reduction for each individual phase matrix. It can be observed that the proposed new Riemann matrix performs exceedingly well than the other matrices which are considered in this experimentation. The proposed new Hadamard matrix offers a PAPR reduction which is nearly the same as that of the Centering matrix. The Centered Hadamard performs better than the original Hadamard and the Centered Riemann provide as a more PAPR reduction than the original Riemann matrix. The proposed new Riemann matrix shows a near elimination of around $8 \mathrm{db}$ to $8.5 \mathrm{db}$ is obtained. In [7], it is stated that the Centering matrix shows the PAPR reduction more than the Riemann (which can also be seen in Figure 2). From this research work, it can be stated that the proposed new Riemann matrix performs even better than the Centering matrix.

The proposed new Riemann matrix was tested for its PAPR performance on a fixed input bit stream by taking the different M-QAM constellations and the IFFT sizes. Four times oversampling of the modulated symb9ols is performed before taking the IFFT. The following table demonstrates the results. An OFDM symbol is generated by using the combinations of the M-QAM and the FFT and the minimum PAPR $(\mathrm{db})$ is found out. It can be observed from the Table 2, that as the IFFT size increases, the PAPR of the OFDM signal reduces by a significant value. For the same IFFT size, if the M-QAM constellation is changed, the PAPR of the particular signal does not undergo any major change.

The OFDM symbols are transmitted through the AWGN channel that is the AWGN noise is added to the transmitted signal. The heftiness of a communication scheme is determined by the recital of the receiver. The BER versus SNR performance for the various phase matrices is compared to that of the unmodified OFDM in Figure 3. The unmodified original OFDM and the SLM with the Hadamard and the Centering matrix present almost the same performance. The BER of the system with the proposed new Riemann matrix can be reduced by applying a high SNR. As the trade of is usually experienced in the communication, here also a trade off in between the BER and the PAPR performance in the proposed scheme is seen.

\section{Conclusion}

In this research paper, a modification in the phase sequence for a conventional SLM scheme is being proposed. The proposed new Riemann phase sequence shows the PAPR reduction to a greater extent as compared to that which is obtained by using the other phase altering 
matrices. It indeed outperforms the already proposed Centering, Riemann and the Hadamard matrices, in terms of amount of PAPR reduction in the OFDM. However, the proposed phase sequences cause an increase in the bit error rate (BER). In order to reduce the BER, a highly powerful error control codes (which have the effect of an increase in the bandwidth) can be used. The use of a higher transmit power can be another alternative for the BER reduction. So, either of the two resources, the signal to noise ratio (SNR) or the bandwidth can be used in order to reduce the BER.

\section{References}

[1] Richard Van Nee, RamjeePrasad, "OFDM for Wireless Multimedia Communication", Artech House Universal Personal Communication Library, Boston, London, pp. 33-37.

[2] Charan Langton, "Intuitive Guide to Principles of Communication, Orthogonal Frequency Division Multiplexing Tutorial", Copyright 2004, Wadsworth, 1993, pp. 123-135.

[3] Tao Jiang and Yiyan Wu, "An Overview: Peak to Average Power Ratio Reduction Techniques for OFDM Signals", IEEE Transactions on Broadcasting, Volume 54, No. 2, June 2008.
[4] D. S. Jayalath and C. Telambura, "SLM and PTS Peak Power Reduction of OFDM Signals Without Side Information", IEEE Transactions on Wireless Communication, Volume 4, No. 5, September 2005.

[5] N. V. Irukulapati, V. K. Chakka and A. Jain, "SLM Based PAPR Reduction of OFDM Signals by using New Phase Sequence", Electronics Letters, $19^{\text {th }}$ November 2009, Volume 45, No. 24.

[6] M. Palanivelan, Dr. Sheila Anand and M. Gunasekaran, "Matrix Based Low Complexity PAPR Reduction in OFDM Systems", IJECT, Volume 2, Issue 2, June 2011.

[7] ThitaphaChanpokapaiboon, PotcharaPuttawanchai and PrapunSuksompong, "Enhancing PAPR Performance of MIMOOFDM Systems by using SLM Techniques with Centering Phase Sequence Matrix", ECTI Association of Thailand-Conference 2011, Communication Systems Wireless/Mobile Communications \& Technologies, Paper ID: 1481.

[8] William Revelle, "Matrix Algebra", Northwestern University January 24, 2007.

[9] Marco Breiling, Stefan H. Muller-Weinfurtner and Johannes B. Huber, "SLM Peak Power Reduction Without Explicit Side Information", IEEE Communications, Volume 5, No. 6, June 2001.

[10] Lajos Hanzo, Raymond Steete and Peter-Marc Fortune, "A Sub Band Coding and 16 QAM System for Mobile Radio Speech Communication", IEEE Transactions on Vehicular Technology, Volume 39, No.4, November 1990. 